\title{
La simulation globale sur modèle réduit des écoulements de convection mixte
}

\section{Overall scale-model simulation of mixed-convection flow}

\author{
Jean-Paul Huffenus \\ Chef des Services Techniques Généraux adjoint \\ Société Neyrpic
}

$\begin{array}{ll}\text { Notations } \\ C & \text { Chaleur spécifique } \\ C f & \text { Coefficient de frottement } \\ D & \text { Dimension géométrique } \\ F r & \text { Nombre de Froude } \\ g & \text { Accélération de la pesanteur } \\ G r & \text { Nombre de Grashof } \\ h & \text { Coefficient d'échange fluide paroi } \\ M s & \text { Nombre de Margoulis } \\ P r & \text { Nombre de Prandtl } \\ P r_{t} & \text { Nombre de Prandtl turbulent } \\ R a & \text { Nombre de Rayleigh } \\ R e & \text { Nombre de Reynolds } \\ S & \text { Surface } \\ T, \delta T & \text { Température ou écart de température } \\ V & \text { Vitesse } \\ \alpha & \text { Diffusivité } \\ \alpha & \text { Diffusivité apparente } \\ \nu & \text { Viscosité cinématique } \\ \nu_{t} & \text { Viscosité turbulente } \\ \rho & \text { Masse spécifique } \\ \delta \rho & \text { Variation de densité } \\ & \end{array}$

\section{Introduction}

Les écoulements de convection mixte se rencontrent fréquemment dans de multiples domaines industriels, ventilation, pollution, réacteurs chimiques, réacteurs nucléaires... Ces écoulements sont caractérisés par l'action simultanée d'effets d'inertie liés à une convection forcée et d'effets de gravité dûs à des différences de densité provoquées par des variations de température ou de concentration de produits en solution. L'étude de ces écoulements présente de nombreuses difficultés et l'ingénieur dispose en réalité de peu de moyens efficaces pour quantifier de façon sûre les phénomènes lors de l'établissement d'un projet donné.

Le recours à une modélisation mathématique demande la résolution simultanée des équations de type Navier-Stokes et de l'équation de la chaleur, le tout accompagné d'une simulation de turbulence convenable pour laquelle les effets de gravité ne sont pas sans apporter un incrément de complexité supplémentaire. Bien que posant encore de gros problémes tant au plan physique qu'au plan numérique, de tels calculs sont aujourd'hui relativement courants. Toutefois ils sont limités :

- à des géométries relativement simples. Ainsi sans parler de tridimensionnel, il existe encore des géométries bidimensionnelles qui doivent être exclues si l'on veut traiter avec soin les phénomènes près de la paroi ;

- à des conditions aux limites n'induisant pas des écoulements de caractère trop fortement instationnaires auquel cas le calcul pour être quelque peu significatif est d'une longueur extrême.

Le modèle réduit présente donc a priori un attrait tout particulier car il n'est gêné ni par la complexité géométrique ni par l'instationnarité des phénomènes.

Toutefois, là encore, la simulation globale, c'est-à-dire la représentation directe à échelle réduite de l'écoulement réel, n'a rien d'évident dans le cas général. Une analyse préliminaire des conditions de similitude et de leur réalisation pratique est indispensable ; elle conduira à mieux cerner les domaines de validité des résultats que l'on peut tirer du modèle et dans les mauvais cas à rejeter l'éventualité d'une telle simulation globale.

En cas d'impossibilité, l'ingénieur ne sera pas totalement démuni. Faute de mieux, il peut essayer de décomposer les phénomènes en cause en les analysant sous divers aspects partiels qui seront peut-être plus 
abordables, soit sur des modèles mathématiques, soit sur des modèles physiques. Par des combinaisons astucieuses de ces derniers, il arrivera souvent à reconstruire plus ou moins le "morceau" de réalité qui l'intéresse. Il faut cependant bien reconnaître que ce genre de démarche reste relativement hasardeux et que les résultats ainsi obtenus ne seront pas toujours probants, laissant place à des incertitudes et à d'innombrables discussions à partir d'éléments plus ou moins subjectifs.

C'est pourquoi la simulation globale lorsqu'elle est réalisable, conserve un réel intérêt. Nous en examinerons ici ses possibilités de façon objective en essayant de conserver à l'analyse un caractère de généralité suffisant pour couvrir la grande majorité des nombreuses applications concernées par ces écoulements de convection mixte.

\section{Conditions générales de similitude}

Il est facile de montrer à partir des équations qui rendent compte des phénomènes que dans le cadre de l'approximation de Boussinesq $(\delta \rho / \rho \ll 1)$, les paramètres de similitude sont les suivants :

Nombre de Reynolds $V D / \nu$ (caractéristique de la convection forcée)

Nombre de Grashof $g \frac{\delta \rho}{\rho} D^{3} / \nu^{2}$ (caractéristique de la convection naturelle)

Nombre de Prandtl $\nu / \alpha$ (caractéristique du fluide)

(ou nombre de Schmidt pour le transfert de masse. Nous supposerons dans ce qui suit que les différences de densité sont d'origine thermique).

Il est non moins facile de voir que dans la grande majorité des cas industriels qui font intervenir de grandes dimensions, toute similitude basée sur le respect de ces trois nombres n'a d'autre issue possible que l'échelle 1 .

S'inspirant d'une démarche classique en hydrodynamique isotherme, on peut utiliser le caractère généralement très turbulent des écoulements en question pour réduire sur le modèle les nombres de Reynolds et de Grashof en continuant toutefois à respecter leur "rapport" qui est un nombre de Froude ou un nombre de Richardson.

$$
F r=R e / \sqrt{G r}=V / \sqrt{g D \delta \rho / \rho}
$$

(Dans tout ce qui suit, on se placera en similitude de Froude ou de Richardson).

On accepte ainsi une certaine distorsion des effets visqueux qui affecte principalement l'écoulement dans les zones pariétales et en particulier le coefficient de frottement $C f$.

Dans les modèles classiques isothermes, cette distorsion n'est pas gênante à condition qu'elle soit limitée à une valeur raisonnable de façon à ne pas affecter sensiblement l'écoulement au sein même du fluide, c'est-àdire hors des zones pariétales. Des données théoriques et d'expérience permettent d'apprécier, pour chaque cas particulier et en fonction de la précision recherchée, ce qu'il est raisonnable d'accepter.
Par contre pour les modèles non isothermes, cette réduction du nombre de Reynolds devient a priori inacceptable pour plusieurs raisons :

a) le frottement joue un rôle essentiel dans la limitation des mouvements de convection naturelle

b) la répartition des températures peut être gravement perturbée :

- au sein du fluide, car les effets de la conduction moléculaire sont exhaltés par la réduction des dimensions géométriques

- au voisinage des parois, pour cette même raison à laquelle s'ajoute les répercussions de la distorsion du champ dynamique évoquée en a)

Il est bien entendu impossible de rattraper tous ces défauts en même temps et de façon rigoureuse. Toutefois, si l'on accepte l'idée d'une simulation de portée limitée, on peut voir qu'il est possible de jouer sur le nombre de Prandtl du fluide modèle pour atténuer les effets néfastes de la réduction de Reynolds.

Un peu schématiquement on peut appuyer cette idée sur des raisonnements du type suivant:

A) les effets de conduction moléculaire peuvent être ramenés à l'échelle des transferts convectifs en conservant le nombre de Peclet $(P e=V D / \alpha)$ ce qui sousentend le choix d'un fluide modèle relativement moins conducteur que le fluide réel et tel que ${ }^{(1)}$ :

$$
\overline{P r}=\overline{R e^{-1}}
$$

B) la distorsion des couches limites est inévitable ; toutefois on doit se fixer comme exigence minimum que les transferts de chaleur à travers les parois soient à l'échelle des transferts convectifs au sein du fluide, c'està-dire que l'on respecte le nombre de Margoulis (ou de Stanton) :

$$
\begin{aligned}
& M s & =h / \rho c V \\
\text { donc } & \overline{M s} & =1
\end{aligned}
$$

Or si l'on admet très sommairement l'analogie de Chilton-Colburn :

$$
M s=\frac{C f}{2} \operatorname{Pr}^{-2 / 3}
$$

avec une variation de $\mathrm{Cf}$ en $\mathrm{Re}^{-1 / 4}$ comme dans la loi de Blasius, on a :

soit

$$
\begin{aligned}
& \overline{M s}=\overline{R e}-1 / 4 \overline{P r}^{-2 / 3}=1 \\
& \overline{P r}=\overline{R e}-3 / 8
\end{aligned}
$$

Ceci revient à peu près à conserver sur le modèle le produit $\operatorname{Pe} \operatorname{Pr}$ (ou $R e \operatorname{Pr}^{2}$ ).

Cette analyse un peu sommaire montre qu'il y a donc avantage à adapter le choix du fluide modèle pour rendre plus tolérable certains effets de la distorsion de Reynolds ; elle laisse par contre prévoir qu'il n'y aura pas a priori unicité de solution puisque dans un cas (A) on recommande de choisir $\overline{P e}=1$ et dans l'autre (B) $\overline{P e} \overline{P r}=1$. Nous allons maintenant revenir plus en détail sur ces points.

(1) On notera $\bar{X}$ le rapport de la grandeur $X$ sur le modèle à la grandeur $X$ sur le réel. 
Similitude dans les zones "éloignées des parois"

La réduction du nombre de Reynolds sur le modèle est précisément limitée pour que, en terme de convection forcée pure, l'écoulement soit encore convenablement représenté dans ces zones "éloignées des parois". Regardons alors ce qu'il advient du champ thermique. Si l'on admet le concept des diffusivités turbulentes scalaires $\left(v_{t}, \alpha_{t}\right)$ on a une diffusivité apparente :

$$
\alpha_{a}=\alpha\left(1+\frac{P r}{P r_{t}} \frac{\nu t}{\nu}\right)
$$

L'équation thermique s'écrit en régime permanent:

$$
V \operatorname{grad} T=\alpha \operatorname{div}\left(\alpha_{a} / \alpha \operatorname{grad} T\right)
$$

soit en utilisant des grandeurs sans dimensions primées $\left(X^{\vartheta}=X / X_{0}\right)$

$$
V^{\prime} \operatorname{grad}^{\prime} T=\frac{\alpha}{V_{0} D_{0}} \operatorname{div}^{\prime}\left(\alpha_{a} / \alpha \operatorname{grad}^{\prime} T\right)
$$

Or si les caractéristiques dynamiques de l'écoulement $V$ et $v_{t} / v$ sont bien représentées, on peut poser $v_{t} / \nu=K R e$ où $K$ est une constante. On a ainsi :

$$
V^{\prime} \operatorname{grad}^{\prime} T=\operatorname{div}^{\prime}\left[\left(\frac{1}{P_{e}}+\frac{K}{p_{r}}\right) \operatorname{grad}^{\prime} T\right]
$$

On en déduit :

1) Si le terme diffusion moléculaire (conduction) est important, on doit respecter une similitude de Peclet, done :

$$
\overline{P r}=\overline{R e}^{-1}
$$

Ceci sera particulièrement indiqué lorsque le fluide réel est très conducteur (métaux liquides) ou lorsque l'écoulement risque de présenter des zones fortement stratifiées dans lesquelles la turbulence est très réduite.

2) Le terme représentant les effets diffusifs turbulents n'impose pas de conditions particulières à condition que $\mathrm{Pr}_{t}$ soit constant. Or ceci n'est vrai que pour

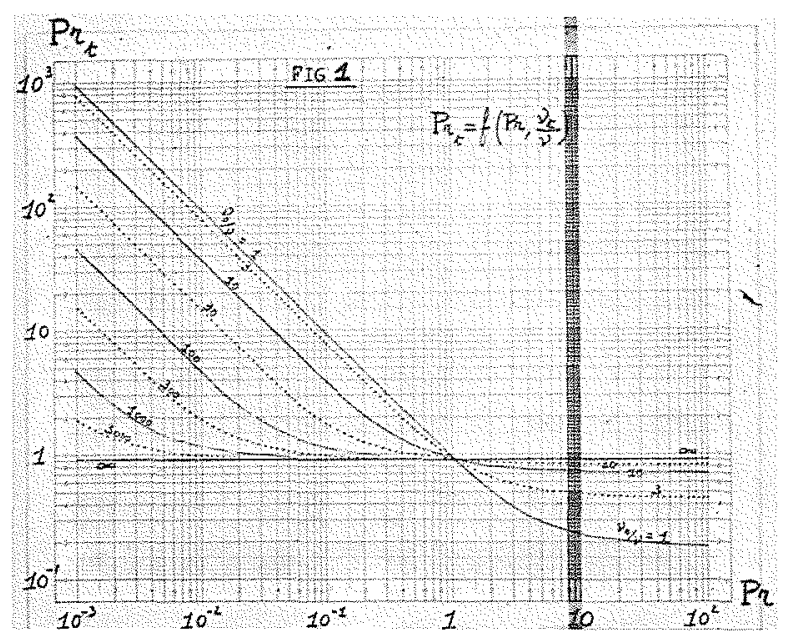

Figure 1. - Variation du nombre de Prandtl turbulent en fonction de $\operatorname{Pr}$ et $\nu_{t} / \nu$.

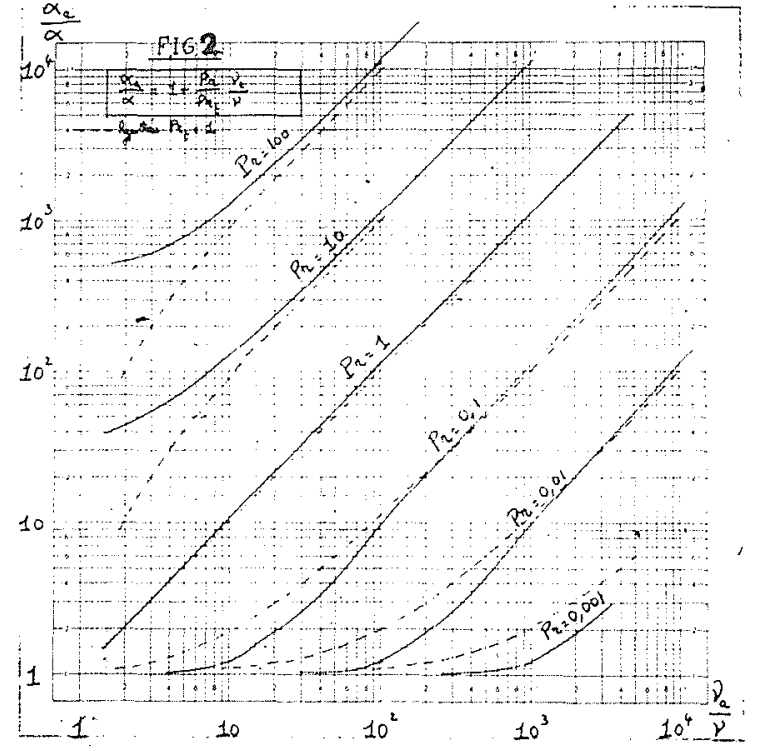

Figure 2. - Variation de la diffusivité apparente rapportée à la diffusion moléculaire en fonction de $\operatorname{Pr}$ et $\nu_{t} / \nu$.

$\nu_{t} / \nu$ assez grand si l'on en juge par exemple par l'expression donnée par A.T. Wassel et I. Catton [1] qui se trouve représentée figure 1. En fait, les variations de $\operatorname{Pr}_{t}$ aux faibles valeurs de $\nu_{t} / \nu$ entraînent des variations de la "constante" $K$ si bien qu'au total la valeur de $K / P r_{t}$ donc de $\alpha_{q} / \alpha$ calculable par (1) (Fig. 2) ne diffère pas notablement de ce que l'on calculerait avec $\operatorname{Pr}_{t}$ constant. Ceci permet donc de négliger a priori l'effet des variations de $\mathrm{Pr}_{t}$ et de considérer les effets diffusifs turbulents comme correctement représentés.

\section{Similitude des phénomènes au voisinage des parois}

\section{Généralités}

La distorsion du nombre de Reynolds ne permet pas d'espérer reproduire sur le modèle les caractéristiques précises des couches limites. Toutefois, il est important de conserver au moins à l'échelle les transferts de chaleur pariétaux $(h S \delta T)$. Or, l'échelle du modèle est essentiellement fixée par les transferts convectifs au sein du fluide $(\rho c V S \delta T)$. Ceci entraîne le respect du nombre de Margoulis.

Pour juger globalement des distorsions que peut entraîner un modèle sur ce point, il est nécessaire de recourir à des corrélations suffisamment générales, en particulier pour ce qui concerne le nombre de Prandtl, de façon à ce qu'elles puissent être appliquées valablement à la fois au modèle et au réel. Ces corrélations ne sont disponibles dans la littérature que pour quelques phénomènes simples du type :

- convection naturelle sur une plaque plane verticale ou sur un cylindre horizontal

- convection forcée sur une plaque plane, dans une conduite ou sur un cylindre.

Nous les appliquerons en nous situant dans le cadre précis d'un fluide réel donné. Trois cas seront envisagés 


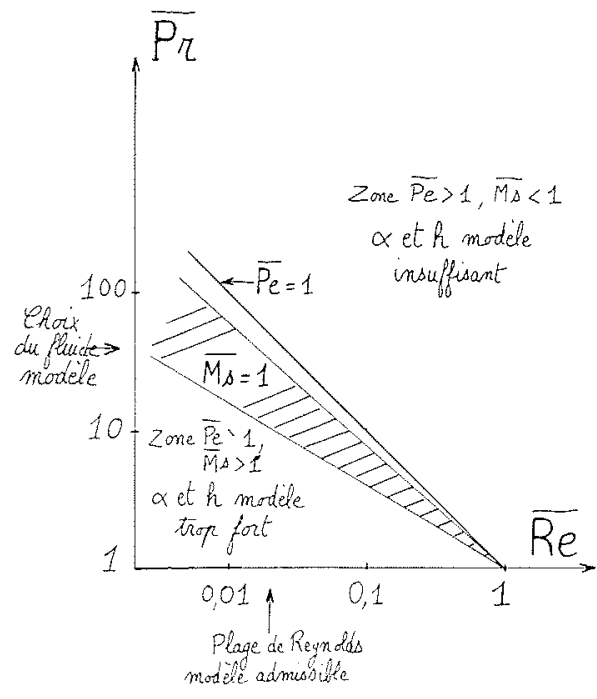

Figure 3. - Diagramme $(\overline{R e}, \overline{P r})$ - délimitation des zones.

selon que ce dernier est l'eau $(P r=5)$, l'air $(P r=0,7)$ ou le sodium liquide $(\operatorname{Pr}=0,005)$. Pour chaque cas, nous établirons un diagramme $(\overline{R e}, \overline{P r})$ permettant de lire directement pour une distorsion de Reynolds donné la distorsion la mieux adaptée du nombre de Prandtl pour remplir un objectif donné. Comme le montre qualitativement la figure 3 , les lignes $\overline{P e}=1$ et $\overline{M s}=1$ délimitent des zones dans lesquelles le modèle présentera :

- une diffusivité $\alpha$ et des coefficients d'échange trop forts si $\overline{P r}$ est faible (égal ou peu supérieur à 1)

- une diffusivité $\alpha$ et des coefficients d'échange trop faibles si $\overline{P r}$ est grand

\section{Convection naturelle}

S.W. Churchill et H.H.S Chu. [2] donnent une corrélation générale pour les plaques planes verticales à température constante :

$$
N u^{1 / 2}=0,825+\frac{0,387 R a^{1 / 6}}{\left[1+(0,492 / P r)^{9 / 16}\right]^{8 / 27}}
$$

pour des valeurs $R a$ allant jusqu'à $10^{12}$ et pour des fluides variés (mercure, eau, air, huile...).

Le rapport $\overline{M s}$ ne s'exprimera de façon simple que dans les deux cas particuliers:

$-R a \sim 0$

Dans ces conditions $\overline{N u}=1$ donc $\overline{M s}=\overline{P e}^{-1}$

$-R a \sim \infty$

$$
M s^{1 / 2}=\frac{0,387 \operatorname{Re}^{-1 / 2} \operatorname{Pr}^{-1 / 2} R a^{-1 / 6}}{\left[1+(0,492 / P r)^{9 / 16}\right]^{8 / 27}}
$$

On peut donc en déduire $\overline{M s}$ sachant qu'en similitude de Froude

$$
\overline{R a}=\overline{R e} e^{2} \overline{P r}
$$

On constate alors que $\overline{M s}=1$ est obtenu pour un couple $(\overline{R e}, \overline{P r})$ tel que :

$$
\left.\overline{\operatorname{Re}} \overline{P r^{2}} \overline{\left[1+(0,492 / P r)^{9 / 16}\right.}\right]^{16 / 9}=1
$$
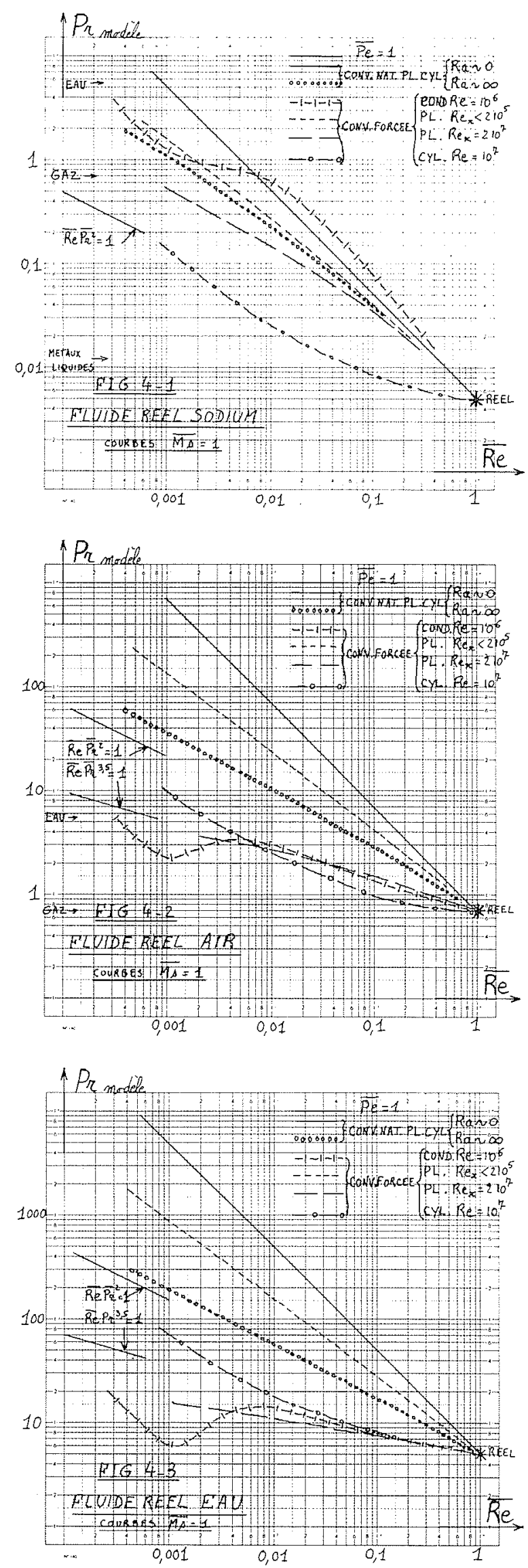

Figure 4. - Diagrammes $(\overline{R e}, \overline{P r})$ pour le sodium (4-1), l'air (4-2) et l'eau (4-3) montrant les courbes $\overline{M s}=1$ pour des écoulements de convection naturelle et forcée. 
Les figures 4 montrent dans le plan $(\overline{R e}, \overline{P r})$ la courbe $\overline{M s}=1$ pour les trois valeurs de Pr réel.

Si l'on considère une corrélation générale relative cette fois-ci à des cylindres à axes horizontaux [3], la courbe $\overline{M s}=1$ se superpose avec les précédentes.

\section{Convection forcée}

L'homogénéité relative des résultats en convection naturelle ne semble pas devoir se maintenir si l'on considère maintenant des écoulements de convection forcée. Plusieurs types d'écoulements sont à considérer :

\section{a) l'écoulement établi en conduite lisse}

La corrélation générale développée dans [4] tenant compte de l'ensemble des régimes laminaire et turbulent a été utilisée. Sous sa forme simplifiée, limitée au régime turbulent, cette corrélation est de la forme :

$$
N u=5,55+\frac{0,0357 \operatorname{Pr}}{\left(1+\operatorname{Pr}^{4 / 5}\right)^{5 / 6}} \frac{R e}{\ln (\operatorname{Re} / 7)}
$$

Appliquée à un nombre de Reynolds réel de $10^{6}$, elle conduit aux courbes $\overline{M s}=1$ des figures 4 .

\section{b) l'écoulement sur une plaque plane}

On considère la corrélation indiquée dans [5] pour les couches limites laminaires :

$$
N u=\frac{0,564 \operatorname{Re}_{x}^{1 / 2} \operatorname{Pr}^{1 / 2}}{\left[1+\left(\frac{P r}{0,0468}\right)^{2 / 3}\right]^{1 / 4}} \text { avec } R e_{x}<510^{5}
$$

Pour les couches limites turbulentes, une application numérique pour $R e_{x}=210^{7}$ a été réalisée sur les bases de la corrélation développée par Kader et Yaglom dans [6]. Une comparaison avec les corrélations indiquées dans [7] montre une légère dispersion entre ces diverses corrélations.

\section{c) l'écoulement transversal sur un cylindre}

La corrélation développée dans [8] a été appliquée pour un nombre de Reynolds du cylindre de $10^{7}$.

\section{Conclusions}

L'examen des figures 4 suggère les conclusions suivantes :

L'augmentation du nombre de Prandtl sur le modèle peut effectivement "compenser" globalement la distorsion entraînée par la réduction du nombre de Reynolds au niveau des échanges pariétaux. Toutefois, on n'observe malheureusement pas un resserrement des courbes vers une similitude unique. Généralement, on tombe sur une règle différente du simple respect du nombre de Péclet :

$$
\overline{\operatorname{Re}} \overline{\operatorname{Pr}}^{n}=1 \quad \text { avec } \quad n>1
$$

$n$ est d'autant plus grand :

- que le fluide réel est à un nombre de Prandtl plus élevé. De ce point de vue l'étude d'écoulements de sodium liquide parait favorisée par une dispersion modérée autour de $n \sim 1,5$
- que les transferts sont sensibles à des phénomènes de convection forcée très turbulents. Il faut noter que les échanges en convection naturelle s'accommodent mieux d'une similitude voisine de celle de Péclet que les échanges en convection forcée.

Bien entendu, les difficultés de réalisation d'une similitude acceptable augmentent avec la distorsion admise sur le nombre de Reynolds.

\section{Représentation des parois sur le modèle}

La non concordance et la dispersion des impératifs à satisfaire pour conserver à l'échelle les transferts à la paroi et au sein du fluide entraînent une qualité très discutable de la simulation. Cet inconvénient majeur peut être réduit grâce à certains artifices dans la réalisation des parois du modèle.

a) L'épaisseur $e$ de la paroi peut être corrigée sur le modèle de façon à ce que le coefficient d'échange global d'un côté à l'autre (incluant donc les deux couches limites fluides) soit grosso modo correct alors que chacun des deux coefficients d'échange des couches limites est distordu. Une telle correction n'est bien entendu acceptable que dans la mesure où cette dernière distorsion est faible. En effet on ne peut en toute rigueur rattraper par un terme fixe (lié à la correction de l'épaisseur), la distorsion d'un terme éminemment variable puisque les coefficients d'échange pariétaux changent d'un point à un autre selon l'écoulement et sont a priori inconnus.

b) Plus intéressante est l'idée d'une rugosité de paroi qui peut servir, par exemple, à relever sur un modèle en similitude de Péclet un coefficient d'échange a priori insuffisant comme le montrent les figures 4 . Ce second artifice permet une correction directe sur la grandeur distordue. Il n'est pas d'ailleurs sans rappeler des artifices analogues bien connus en hydraulique puisque les modèles à surface libre (eux aussi en similitude de Froude) comportent souvent au fond des rugosités artificielles pour retrouver une pente correcte des lignes d'eau, la réduction du nombre de Reynolds ayant modifié les coefficients de frottement.

De plus, nous avons observé sur les figures 4 que les modèles qui s'écartent le plus de la similitude de Péclet (donc ceux qui ont le plus besoin d'être "corrigés") sont ceux qui représentent des phénomènes réels dans des liquides à relativement haut nombre de Prandtl s'écoulant principalement en convection forcée. Or sans que l'on puisse disposer de données irréfutables à cet égard, il semble justement que le relèvement du coefficient d'échange dû à l'effet de rugosité de la paroi soit surtout sensible dans ces dernières conditions. L'artifice rugosité tendrait donc à resserrer un peu les courbes $\overline{M s}=1$ des figures 4 . La vérification de cette assertion se heurte toutefois à un manque d'informations disponibles dans la littérature, en particulier sur l'effet de rugosité en convection naturelle.

c) Les caractéristiques thermiques des matériaux disponibles pour réaliser le modèle obligent parfois à distordre l'épaisseur de la paroi qui ne se trouvera plus à l'échelle géométrique. Ceci peut amener des difficultés 
pour représenter certaines formes à faible rayon de courbure. Par ailleurs, on ne retrouvera que très rarement satisfaites les conditions qui seraient à réaliser pour conserver une simulation correcte des aspects thermiques instationnaires (similitude de Fourier). Ceci élimine certaines applications portant sur l'examen de régimes transitoires et demande à être examiné de plus près lorsqu'on s'intéresse à des écoulements instationnaires où des fréquences assez basses peuvent se manifester.

\section{Applications}

Les difficultés qui ressortent de ce qui précède et qui rendent l'existence d'une solution satisfaisante particulièrement incertaine, ne sont pas heureusement toutes présentes avec le degré d'acuité maximal dans les problèmes industriels de convection mixte.

- Certains problèmes simples ne font pas intervenir les échanges de chaleur à travers des parois et bénéficient donc des conclusions simples développées plus haut pour les zones éloignées des parois (similitude de Froude seul ou de Froude-Péclet si nécessaire) Cf. $[9,10]$.

- Dans le cas où l'on doit prendre en compte les phénomènes au voisinage de la paroi, on peut parfois négliger l'effet de conduction et se ramener à une similation approchée basée sur les développements cidessus conduisant aux figures 4 (similitude de Froude-Margoulis).

- Quand ceci n'est pas possible, le compromis devient plus délicat et le recours à des artifices peut être nécessaire pour "marier" Froude, Péclet et Margoulis.

\section{Conclusion}

L'étude des possibilités de simulation globale montre que l'on peut effectivement éviter par ces modèles des démarches indirectes où les enchaînements ne sont pas toujours convaincants et d'une rigueur irréprochable. Toutefois, comme on l'a vu, les difficultés peuvent être très grandes ; les latitudes de réduction du nombre de Reynolds seront généralement plus réduites qu'en convection forcée pure ; enfin on peut être amené ainsi à des technologies de modèle réduit un peu inhabituelles. C'est pourquoi la solution de la simulation globale n'est pas toujours évidente si l'on désire conserver une certaine rigueur.

Pour échapper aux difficultés, la tentation est grande de simplifier plus avant en tolérant une plus grande distorsion des phénomènes. Il $\mathrm{y}$ a là un réel danger, car rien n'est plus trompeur que l'observation sur un modèle à échelle réduite de phénomènes distordus ; tant il est vrai que le plus averti finit à la longue par prendre inconsciemment ce qu'il aperçoit pour un reflet très approché de la réalité.

\section{Bibliographie}

[1] REYNOLDS A.J. - The prediction of turbulent Prandtl and Schmidt numbers. Int. Journ. of heat and mass transfer. Vol. $18-1975$.

[2] CHURCHILL S.W., CHU H.H.S. - Correlating equations for laminar and turbulent free convection for a vertical plate Int. Journ. of heat and mass transfer, Vol. 18 - 1975.

[3] CHURCHILL S.W., CHU H.H.S. - Correlating equations for laminar and turbulent free convection from a horizontal cylinder. Int. Journ. of heat and mass transfer, Vol. 18 1975.

[4] CHURCHILL S.W. - Comprehensive correlating equations for heat mass and momentum transfer in fully developped flow in smooth tubes.Industrial and Engineering Chemistry Fundamentals, Vol $16 \mathrm{n}^{\circ} 1-1977$

[5] CHURCHILL S.W., USAGI R. - A general expression for the correlation of rates of transfer and other phenomena A I ch E-Journal, 18-1972.

[6] KADER B.A., YAGLOM AM. - Heat and mass transfer laws for fully turbulent wall flows Int. Journal of heat and mass transfer, Vol. $\mathrm{n}^{\circ} 15-1972$.

[7] CHURCHILL S.W. - A comprehensive correlating equations for forced convection from flat plates $A$ I Ch E journal, Vol $22 \mathrm{n}^{\circ} 2-1976$.

[8] CHURCHILL S.W. BERNSTEIN M. - A correlating equation for forced convection from gases and liquids to a circular cylinder in cross flow. Trans ASME - Journal of heat transfer Vol. 99 - 1977.

[9] LEROUGE B., HUFFENUS JP., LAMBERT P. - Les remontées d'eau active dans la piscine du réacteur Osiris $L a$ Houille Blanche $\mathrm{n}^{\circ} 8-1968$

[10] DAUBERT A., HUFFENUS J.P. - Méthodes d'approches pour les problèmes de convection mixte dans les métaux liquides $-14^{e}$ Congrès $A I R H$ - Sept. 71. 


\section{Discussion}

Le Président remercie M. HUFFENUS et ouvre la discussion.

M. BOULOT. - Je crois que M. HUFFENUS a bien distingué les cas où le transfert à la paroi intervient dans le problème traité et le cas où le transfert n'intervient pas. Cette distinction est très importante, parce qu'il existe des problèmes où les transferts à la paroi n'interviennent pas. A ce moment-là, on dispose de plus de facilité pour traiter le problème à l'intérieur de la masse de sodium.

Il faut distinguer deux cas :

- le cas où l'on a des risques de stratification : je crois que le problème est très difficile à traiter, puisque, en toute logique, on doit respecter la similitude de Péclet, mais aussi celles de Reynolds et de Froude, ce qui est impossible ;

- le cas où l'on n'a pas de risque de stratification : on peut faire beaucoup de choses, à condition de ne pas descendre trop en nombre de Reynolds; si on traite un problème de sodium et si on a une bonne turbulence, il n'est même pas certain qu'on ait à respecter la similitude de Péclet.

Il faut respecter une similitude de Froude en espérant qu'on n'aura pas de stratification importante, auquel cas la solution trouvée n'est certainement pas la bonne.

M. HUFFENUS. - C'est exact. Quand on a une stratification, il faudrait, en toute rigueur, non seulement respecter le nombre de Richardson et le nombre de Peclet, mais respecter aussi le nombre de Reynolds. C'est un voeu pieux. Mais si, malgré tout, on réduit relativement peu le nombre de Reynolds, on a néanmoins une chance d'apercevoir un phénomène qui ait un sens.

Je crois que, finalement, tout le problème de ces modélisations est de bien savoir quelle est la réduction de Reynolds admissible. Nos "anciens" nous ont laissé en isotherme une pratique relativement importante des réductions admissibles du nombre de Reynolds pour les phénomènes de convection forcée.

Il reste néanmoins un très gros point d'interrogation en convection mix te et je crois que la précaution la plus élémentaire consiste en effet à réduire le moins possible le nombre de Reynolds.

M. REMENIERAS, - Les pionniers de l'utilisation des modèles réduits hydrauliques classiques ont parfois tenté de vérifier expérimentalement les lois de similitudes alors utilisées et de préciser leurs limites pratiques d'emploi. A cet effet, ils comparaient les résultats fournis par deux ou trois modèles d'un même système assez simple ne différant que par leur échelle (choisie de plus en plus petite). Une vérification moins cô̂teuse était dans certains cas recherchée en augmentant - autant que les installations disponibles le permettaient - les débits circulant dans le mođèle afin de tester sa "sensibilité" aux variations du nombre de Reynolds. Une approche un peu analogue ne devrait-elle pas être tentée - à titre de recherche fondamentale - pour les modèles évoqués par M. HUFFENUS ?

M. HUFFENUS. - C'est possible, mais je pense qu'à cette époque il y avait probablement plus d'argent qu'aujourd'hui pour étudier un problème donné. Aujourd'hui, on se concentre sur un même modèle et il est hors de question d'en faire trois ou quatre pour étudier la même chose ; pourtant ce serait parfois souhaitable, au moins de temps en temps, et celà aiderait à une interprétation plus clairvoyante des résultats.

Le Président. - Nos "anciens" faisaient plusieurs tentatives avec plusieurs échelles différentes, et si ces tentatives donnaient toutes le même résultat, ils considéraient qu'il y avait des chances pour que ces résultats soient exacts ; ce n'était qu'une présomption et pas une démonstration.

Je remercie M. HUFFENUS. 